\title{
THE DIAPHRAGMATIC FLAP: A MULTIUSE MATERIAL IN THORACIC SURGERY
}

Tommaso Claudio Mineo, MD

Vincenzo Ambrogi, MD
Background: The use of diaphragmatic pedicle flaps for reconstructive procedures in thoracic surgery is not very popular. Nevertheless, it provides considerable advantages. Methods: Our experience covers 10 years (1987-1997) with a total of 25 patients in whom the diaphragmatic flap was used for different purposes. In 6 patients we used the diaphragmatic flap to protect the bronchopleural fistula at its early onset, which was not beyond 12 hours from the clinical diagnosis. We performed prophylactic suture protection after neoadjuvant therapy in 9 high-risk patients who underwent pneumonectomy and in 2 who underwent sleeve lobectomy. Postpneumonectomy pericardial defect repair was performed in 4 patients. In another 4 patients the diaphragmatic flap was used after spontaneous $(n=2)$ and iatrogenic $(n=2)$ lesions of the esophagus after 24 to $\mathbf{7 2}$ hours. Results: No perioperative mortality was recorded. Complications were mainly related to the severe preoperative conditions of the patients: arrhythmia, respiratory insufficiency, and empyema. We report only 2 cases of minimal persistent bleeding from the chest tube, which spontaneously ceased. For those patients who survived for more than 1 year $(n=11)$, no diaphragmatic hernias were recorded. Bronchopleural fistulas and pericardial defects healed in all instances. The diaphragmatic flap was also effective in bronchopleural fistula. A late fistula caused by cancer relapse at the bronchial stump developed in only one patient. Excellent repair was achieved in all patients with esophageal lesions. Conclusions: We conclude that the diaphragmatic flap can be considered a practical, safe, and redundant material particularly indicated for defect or fistula closure and for suture line protection in the thoracic cavity. (J Thorac Cardiovasc Surg 1999;118:1084-9) n 1948, Petrovsky ${ }^{1}$ reported the use of diaphragmatic muscle grafts for reconstructive work in both the thorax and the abdomen. Diaphragmatic flaps have been successfully used to reinforce esophageal perforations and to close chest wall defects for more than 30 years. $^{2}$ In 1968, Brewer and Gazzaniga ${ }^{3}$ proposed phrenoplasty as a method for the management of pleural dead space after pulmonary resection. Elsewhere, we have already described a technique for bronchial stump cov-

From the Department of Thoracic Surgery, Tor Vergata University, Rome, Italy.

Received for publication May 25, 1999; revisions requested July 8, 1999; revisions received Aug 4, 1999; accepted for publication Aug 11, 1999.

Address for reprints: Tommaso C. Mineo, Cattedra di Chirurgia Toracica, Università di Roma Tor Vergata, Ospedale S.Eugenio, p.le Umanesimo 10, 00144 Rome, Italy.

Copyright (C) 1999 by Mosby, Inc.

0022-5223/99 $\$ 8.00+0 \quad \mathbf{1 2 / 1 / 1 0 2 0 7 9}$ erage using the diaphragmatic flaps. ${ }^{4}$ Goldstraw and $\mathrm{Jiao}^{5}$ recently used the diaphragmatic flap in pericardial repair after extensive intrapericardial resection. On the other hand, a wider experience is reported in buttressing suture of spontaneous and iatrogenic esophageal lesions. ${ }^{1,6-10}$ The diaphragmatic pedicle flap has already been used in the treatment of uncomplicated achalasia. ${ }^{1,11}$ Nevertheless, criticism about the use of the diaphragmatic flap for reconstructive purposes still persists.

In this article we present our experience on the use of the diaphragmatic flap in thoracic surgery. Technique of preparation, practical applications, and long-term results are described.

\section{Patients and methods}

Technique. The technique of harvesting is shown in Fig 1. The blood supply is provided by the phrenic artery, which flows along the inferior surface of the muscle. The flap is prepared by cutting the diaphragm in a $\mathrm{U}$ or $\mathrm{V}$ shape and folding 
it along the uncut margin, which works as a hinge. The diaphragm is first divided along the designed posterior margin, starting dorsally from the aortic hiatus, to save the origin of the phrenic artery and explore its territory. As an adjuvant maneuver, we normally introduce a cold-light source inside the abdomen through the initial phrenotomy. For this purpose, we use a flexible fiberoptic cable (3280 Omni-tract Surgical, St Paul, Minn). By means of translucency, the major branches of the phrenic artery are clearly visualized, and the flap preparation is conveniently performed. We also find this technique helpful to avoid hepatic and splenic lesions and to divide peritoneal plications. The length of the flap is never measured; we just make an estimate. As soon as the length of the flap is deemed suitable for the surgical purpose, the direction of the incision is inverted ventrally to draw an arch of variable sharpness. We normally mark the keystone of the flap with a radiopaque clip to facilitate radiographic localization. To provide adequate and vital flap, the width of the base should be approximately one quarter of the entire length. The flap is tailored to be rotated inside the chest cavity, avoiding torsion on the pedicle and allowing sufficient motility and low tension of the remaining muscle. The flap can reach any district of the hemithorax and allow wrapping of the main bronchus; in this specific case, we took into account an additional $2 \mathrm{~cm}$ of length.

The diaphragmatic defect is normally closed by nonresorbable interrupted sutures. Peripheral vascularization can provide enough supply to the residual diaphragm, and its healing does not represent a problem. Unless involved in the neoplasm, the main branches of the phrenic nerve are usually preserved during the flap preparation. The area involved in dissection (Fig 1) comprises posterior branches of the nerve, the interruption of which may allow sufficient motility to the muscle. Postoperative motility was evaluated by radiologic studies at 6 and 12 months after the operation, including fluoroscopy and plain chest radiography at maximal inspiration and expiration. Furthermore, pulmonary function tests were usually accomplished before and 1, 3, 6, and 12 months after the operation.

Patients. From March 1987 to December 1997, diaphragmatic flaps were used in a total of 25 patients for different purposes: early closure of bronchopleural fistula $(n=6)$, bronchial anastomosis after sleeve lobectomy $(n=2)$ or postpneumonectomy bronchial stump protection $(n=9)$, pericardial defect repair $(n=4)$, and closure of esophageal spontaneous $(\mathrm{n}=2)$ and iatrogenic $(\mathrm{n}=2)$ injuries. Clinical data and operative details for every patient are summarized in Table I.

Bronchopleural fistula closure. The pedicle diaphragmatic flap was adopted for early closure of bronchopleural fistula, as mentioned in a previous report. ${ }^{4}$ To date, we treated a total of 6 patients within 12 hours after diagnosis. Fistula diagnosis was made at bronchoscopy soon after the onset of suggestive symptoms or signs. The interval between the first operation and fistula diagnosis ranged from 4 to 15 days. All patients had been operated on for lung cancer, 3 on the right

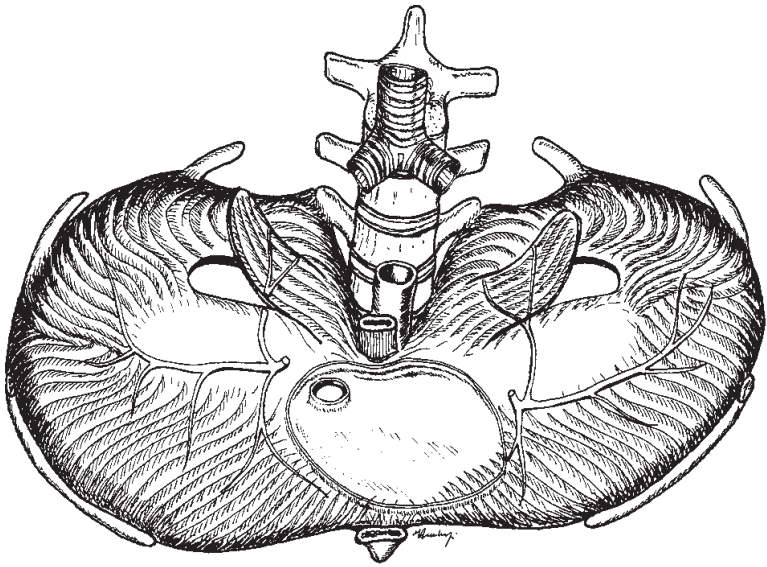

Fig 1. Diaphragmatic flap preparation and its relation with the phrenic nerve. The flap is tailored on the right and left leaflet, with the basis in proximity of the aortic hiatus.

and 3 on the left side, and all but 1 of them had undergone pneumonectomy. The bronchial stump had been handsewn in 3 patients and stapled in 3 others. One patient underwent preoperative radiation therapy, and another had diabetes.

Minimal dissection was usually performed, and the bronchial stump was never dissected out or reshortened. For small fistulas, primary reclosure was accomplished by enclosing with the same interrupted suture as used in the bronchial wall and the diaphragmatic flap. In the case of wide defect, the flap is also adapted as a plug inside the bronchus, which was not tightly closed. The flap was then fixed at the edges by a few nonresorbable sutures to the neighboring vital tissues, avoiding tension or ischemic damage (Fig 2). After bronchial stump testing, the suture line was washed with antibiotic solution. Finally, a chest tube was inserted into the residual thoracic cavity, and it was removed in a similar fashion as after an uncomplicated pneumonectomy or lobectomy.

Prophylactic bronchial suture or anastomosis protection. We used a pedicle diaphragmatic flap as protection in 9 patients with lung cancer (squamous cancer, $\mathrm{n}=6$; large cell cancer, $n=3$ ) who underwent pneumonectomy, 4 on the right and 5 on the left side, and in 2 who underwent sleeve left lower and right upper lobectomies, respectively. All these patients had neoadjuvant therapy because of centrally located T3 $(n=6)$ and T4 $(n=3)$ tumors, and the probability of a postoperative bronchopleural fistula was deemed very high. The technique of harvesting was similar to that described above; in the case of sleeve lobectomy, the size of the flap had to be adjusted to the dimensions of the residual lobe to not interfere with ventilatory dynamics. After pneumonectomy, a redundant flap was used to cover the bronchial stump. In those patients we normally used an interrupted nonresorbable 2-0 suture with double needle stitches for closing the main bronchus. Before knotting the ends, we transfixed the flap in 2 different symmetric points. After this step, we tightened the sutures by adjusting the flap over the suture in a hoodlike 
Table I. Clinical and operative details of the patients

\begin{tabular}{|c|c|c|c|c|c|c|c|c|}
\hline $\begin{array}{l}\text { Patient } \\
\text { No. }\end{array}$ & Age (y) & $\operatorname{Sex}$ & Procedure & $\begin{array}{l}\text { Operative } \\
\text { delay }(h)\end{array}$ & Complications & $\begin{array}{c}\text { Hospital } \\
\text { stay }(d)\end{array}$ & $\begin{array}{c}\text { Follow-up } \\
(\text { (mo })\end{array}$ & Status \\
\hline 1 & 46 & M & BPF closure & 12 & None & 13 & 20 & Dead \\
\hline 2 & 56 & M & BPF closure & 6 & None & 31 & 38 & Alive \\
\hline 3 & 64 & M & BPF closure & 6 & None & 17 & 71 & Alive \\
\hline 4 & 71 & M & BPF closure & 12 & None & 21 & 16 & Dead \\
\hline 5 & 66 & M & BPF closure & 6 & None & 23 & 18 & Dead \\
\hline 6 & 70 & M & BPF closure & 12 & None & 15 & 6 & Alive \\
\hline 7 & 56 & M & BS protection & - & None & 13 & 10 & Dead \\
\hline 8 & 67 & $\mathrm{~F}$ & BS protection & - & None & 14 & 54 & Alive \\
\hline 9 & 59 & M & BS protection & - & None & 12 & 13 & Alive \\
\hline 10 & 55 & M & BS protection & - & None & 14 & 9 & Dead \\
\hline 11 & 78 & M & BS protection & - & Myocardial infarction & 28 & 7 & Dead \\
\hline 12 & 66 & $\mathrm{~F}$ & BS protection & - & None & 12 & 28 & Alive \\
\hline 13 & 64 & $\mathrm{~F}$ & BS protection & - & None & 13 & 21 & Alive \\
\hline 14 & 69 & M & BS protection & - & Recurrence and late fistula & 13 & 10 & Dead \\
\hline 15 & 70 & M & BS protection & - & Pulmonary edema & 25 & 12 & Alive \\
\hline 16 & 65 & M & BA wrapping & - & None & 21 & 10 & Alive \\
\hline 17 & 71 & M & BA wrapping & - & None & 16 & 12 & Dead \\
\hline 18 & 65 & M & PD repair & - & Arrhythmia & 21 & 9 & Dead \\
\hline 19 & 61 & $\mathrm{~F}$ & PD repair & - & None & 15 & 34 & Alive \\
\hline 20 & 77 & $\mathrm{~F}$ & $\mathrm{PD}$ repair & - & Arrhythmia & 25 & 8 & Alive \\
\hline 21 & 73 & M & PD repair & - & None & 12 & 35 & Dead \\
\hline 22 & 56 & M & ESL repair & 72 & Mediastinitis & 69 & 18 & Alive \\
\hline 23 & 34 & $\mathrm{~F}$ & ESL repair & 24 & None & 57 & 34 & Alive \\
\hline 24 & 45 & M & EIL repair & 24 & None & 15 & 128 & Alive \\
\hline 25 & 59 & M & EIL repair & 36 & None & 38 & 88 & Alive \\
\hline
\end{tabular}

$B P F$, Bronchopleural fistula; $B S$, bronchial suture; $B A$, bronchial anastomosis; $P D$, pericardial defect; $E S L$, esophageal spontaneous lesion; $E I L$, esophageal iatrogenic lesion.

fashion (Fig 3). After a sleeve resection, we simply wrapped the anastomosis with one loop of the pedicle flap, which is long enough to fit this purpose. To secure it in place, we considered it sufficient to calibrate the diameter of the loop at the bronchial anastomosis and hold it in place with 2 or 3 flap-toflap resorbable sutures, avoiding further piercing of the bronchus (Fig 4).

Pericardial defect repair. Repair of a postpneumonectomy pericardial defect was performed by using a pedicle diaphragmatic flap in 4 patients. All patients had phrenic nerve involvement with a concomitant palsy of the right hemidiaphragm. A wide flap was usually available, and its size always covered the defect without inducing tension on the superior vena cava and the right atrium. After sewing the flap to the defect edge, we were also able to protect the bronchial stump in 3 patients who had received neoadjuvant therapy.

Esophageal injuries. The diaphragmatic flap was used as an emergency procedure after spontaneous postemetic $(\mathrm{n}=2)$ and iatrogenic $(n=2)$ lesions of the esophagus. The operation was undertaken after a minimum of 24 hours and a maximum of 72 hours (mean, 39.0 hours) from the time of the clinical diagnosis. All lesions were located in the left wall of the distal esophagus. Iatrogenic lesions were caused by pneumatic dilatation for esophageal achalasia and for the removal of a dental plate that was accidentally swallowed. The size of the rent was 5 and $7 \mathrm{~cm}$ for spontaneous lesions and 4 and $9 \mathrm{~cm}$ for iatrogenic lesions, respectively. All lesions were approached through a posterolateral left thoracotomy. The harvesting technique was analogous to that described above. After accurate washing and lavage with sterile saline solution and antibiotics, the lesion was exposed. Mucosal margins of the rupture were isolated and sutured with interrupted nonabsorbable 3-0 sutures. Thereafter, the muscle layer was reconstructed by the interposition of the pedicle flap to protect the mucosal suture and to avoid wall tension (Fig 5). In the case of achalasia, myotomy was extended into the abdomen beyond the gastroesophageal junction through the same incision performed for flap preparation. Two double-lumen drainage tubes were routinely inserted to allow for postoperative lavage.

\section{Results}

No perioperative mortality was recorded. Complications were mainly related to the severe preoperative conditions of the patients (ie, arrhythmia, respiratory insufficiency, and mediastinitis) and are summarized for each patient in Table I. We found only 2 patients with minimal but prolonged bleeding from the chest 


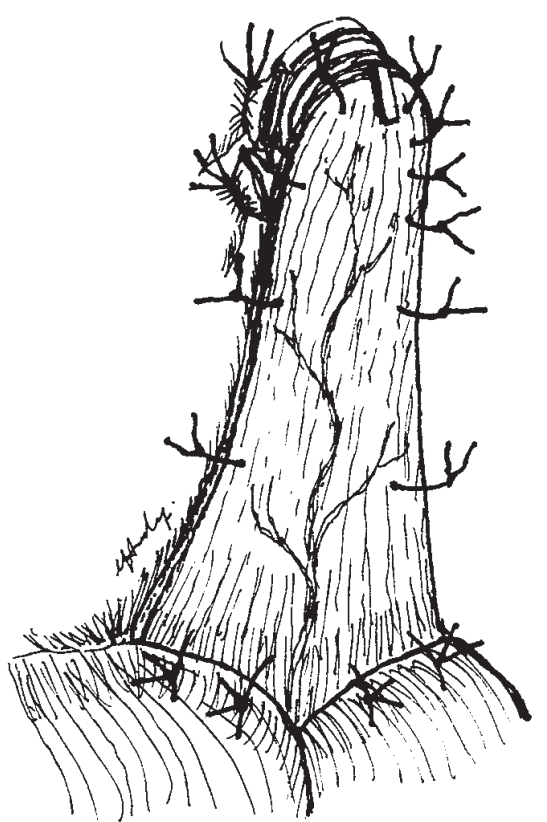

Fig 2. Diaphragmatic flap fixation of the left bronchial stump to cover the fistula.

tube, which spontaneously ceased. No major complications related to the phrenoplasty itself were recorded.

The diaphragmatic defect healed quickly, and no cases of diaphragmatic hernia were noted in our series. For those patients who survived more than 1 year $(\mathrm{n}=$ 11), no diaphragmatic hernias were recorded.

In the group with postpneumonectomy bronchopleural fistulas, the closure was always rapid and effective. Patients were discharged after a mean of 21.0 days (range, 13-31 days) after the fistula operation (Table I). A 3-month postoperative bronchoscopic survey showed bronchial healing in all instances. Three patients died of disseminated disease within 20 months after the operation. The other 3 patients are still alive and well after 6 , 38, and 71 months, respectively (Table I).

The use of a diaphragmatic flap was also effective in prevention of a bronchopleural fistula. Postoperative complications were minimal in all patients. Healing of the bronchial stump and bronchial anastomosis was surveyed by endoscopy and was always satisfactory. A late fistula caused by cancer relapse at the bronchial stump developed in only 1 patient. Neither of the 2 patients who underwent sleeve lobectomy had anastomotic stricture after a 10-month period of follow-up.

All pericardial defects healed promptly and without sequelae. On chest radiography, no patient had radiologic signs of heart herniation or important mediastinal shift.
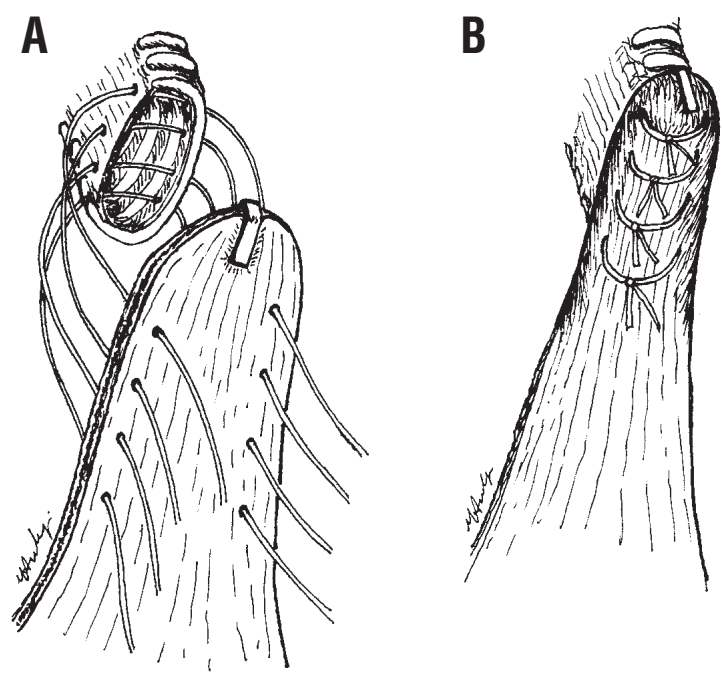

Fig 3. A, Postpneumonectomy right bronchial stump protection. The suture is accomplished with interrupted double-needle stitches. Before knotting the extremities, the flap is transfixed in 2 different symmetric points. B, Thereafter, the stitches are tightened, adjusting the flap over the suture in a hoodlike fashion.

Excellent repair was achieved in all patients with esophageal lesions. The mean hospital stay was 44.75 days (range, 15-69 days). No major discomfort on swallowing or radiologic stenosis were recorded at 1 year after the operation. The patient with achalasia had a significant improvement in deglutition after the Heller myotomy.

As far as the specific function of the diaphragm is concerned, because of lack of preoperative studies, the obvious loss of pulmonary parenchyma, or both, preoperative and postoperative comparative studies were impossible. Examination with imaging techniques demonstrated a mean excursion of $4.3 \mathrm{~cm}$ at phrenic center level in those patients with a residual lobe or an entire lung in place. No effect on ventilatory dynamic or paradoxic motion were found in those patients who underwent pneumonectomy or had phrenic paralysis. Postoperative function tests demonstrated normal and constant flow values (forced vital capacity and forced expiratory volume in 1 second) in those patients who had esophageal repair.

\section{Discussion}

The diaphragm was recently defined by Fell ${ }^{12}$ as "the second most important muscle after the heart." On this basis, some concerns about the use of so important a structure for plastic or reconstructive purposes may arise and persist. Nevertheless, the use of the diaphrag- 


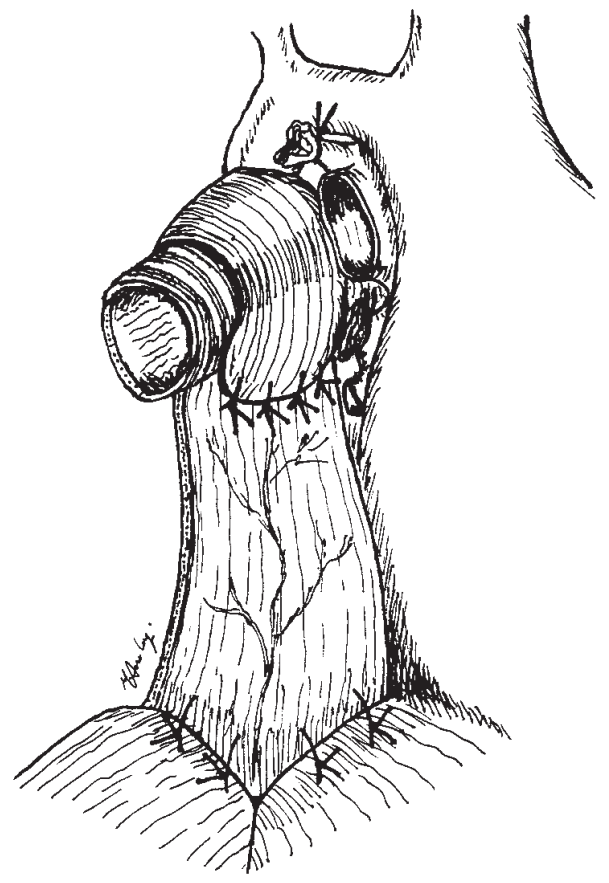

Fig 4. Diaphragmatic wrapping of the bronchial anastomosis after a sleeve upper right lobectomy.

matic flap in thoracic surgery has been documented by several reports. ${ }^{1,3-8,10,11}$ The development of vascular pedicle technique ${ }^{13-15}$ has allowed the intrathoracic transposition of a number of flaps (ie, serratus, intercostal, omental, pectoralis, latissimus, pleural, and pericardial). From a biologic point of view, the effectiveness of pedicle vascular flaps has been demonstrated in microscopic studies showing early neoangiogenesis. ${ }^{13,14}$ These pedicles protect the bronchial structures during the critical revascularization period, which takes 3 to 4 weeks, ${ }^{14}$ and are able to induce neoangiogenesis. ${ }^{16}$ Specific studies on neoangiogenesis after diaphragmatic flap protection are not available at the moment. Nevertheless, endoscopic findings allow us to consider the healing process rapid and uncomplicated. The flap can provide adjunctive properties, such as antiseptic activity and pleural space reduction, because of its bulky and amorphous shape. ${ }^{16}$ In potentially infected or high-risk cases for infection, it is advantageous to have suitable material to cover the defect, to buttress the suture line, and to supply it with healthy and well-vascularized tissue. ${ }^{9}$

Unfortunately, all pedicled flaps require specific and time-consuming preparation, and it is better to foresee their use before entering the pleural space. We reported a previous comparative experience with intercostal muscle in the treatment of early bronchopleural fistula, ${ }^{17}$ but

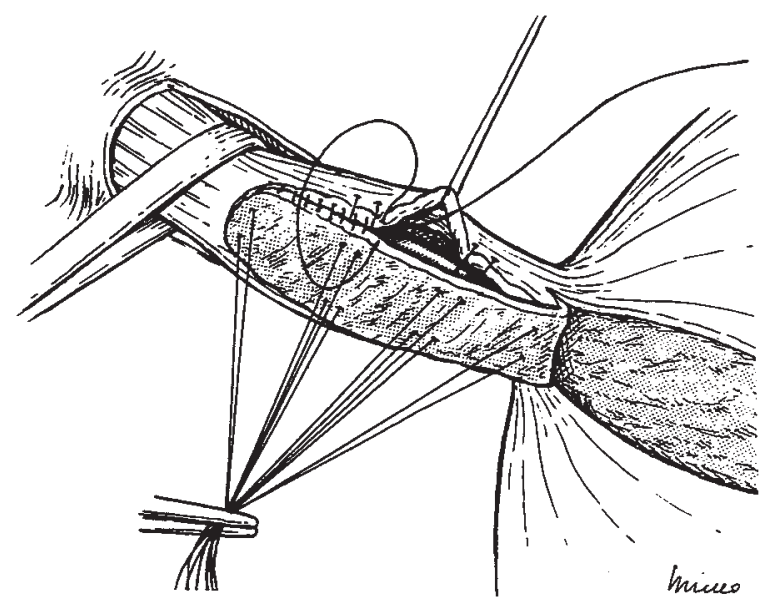

Fig 5. Repair of a lesion sited in the lower third of the esophagus. The diaphragmatic flap is rotated to cover the esophageal tear and stitched to the muscle layer by a double line of interrupted and continuous sutures.

the diaphragmatic flap was more effective. We prefer the diaphragmatic flap for several reasons, even in prophylactic procedures on the bronchus, pericardium, and esophagus. First, there are advantages in the basic technical aspect; the flap is usually easy to prepare, to rotate, and to adapt to the purpose. It is thick enough, resistant to necrosis because of the extensive blood supply to the diaphragm, and immobile once denervated. A long flap can easily be tailored, and it can reach any area of the thoracic cavity without torsion or tension. It is practical and readily available without changing the position of the patient or performing supplementary cutaneous incisions. In the case of pericardial defect, it is sufficiently large to close any defect, and because it is autologous, it is preferable to any synthetic material. Feasibility of the technique can be compromised only by the intrinsic conditions of the diaphragm (eg, fibrosis, scant blood supply, and inflammation).

Finally, we do not consider the opening of the peritoneal cavity during phrenoplasty to represent a real risk of propagating infection to the abdomen. No peritonitis was recorded in our series. Furthermore, no cases of abdominal contamination were reported by Puskas and colleagues ${ }^{15}$ and Kamei and colleagues ${ }^{18}$ in a large series of patients treated by omentum transposition in the hemithorax.

We also found supplementary functional advantages caused by the phrenoplasty itself. As a minor functional effect after phrenoplasty, we noted the decrease of motility of the diaphragmatic posterior leaflet with subsequent reduction of the costophrenic sinus. This may 
allow for an easier re-expansion of the residual upper lobe in case of lower lobectomy, shortening the period of air leakage from an incomplete fissure.

A major matter of interest with regard to phrenoplasty is on the plication effect. Diaphragmatic plication has been successfully proposed in cases of unilateral phrenic palsy ${ }^{19}$ or after pneumonectomy. ${ }^{20}$ Indeed, the loss of an entire lung can provoke several problems in the ventilatory dynamics because of mediastinal instability, and these may be enhanced by the concomitant phrenic nerve lesions caused by cancer invasion. ${ }^{21}$ The closure of the diaphragm subsequent to flap harvesting decreases the muscle surface and reduces its paradoxical movement. In this way phrenoplasty has the same functional effect of diaphragmatic plication and thus may positively affect respiratory function. The plication improves the tolerance to pneumonectomy in those patients with predicted poor residual function. On this basis, phrenoplasty should be considered a supplementary therapeutic device rather than a contraindication.

When dealing with potentially infected lesions, such as bronchopleural fistula and esophageal disruption, our policy allows for early repair, with the exception of esophageal lesions underlying malignant disease. In our experience the operation should not be performed beyond 12 to 24 hours after immediate chest drain insertion. When the reintervention is delayed and the residual cavity is infected, debridement is more difficult, and the failure rate increases exponentially, even if the diaphragmatic flap was successfully used for primary reclosure in an infected field. ${ }^{9}$ Once the decision of repairing has been made, we consider the diaphragmatic flap to be the first choice for repair of lesions located in the middle and lower part of the pleural cavity. In dealing with esophageal ruptures, we still use the Grillo pleural flap. We prefer the diaphragm whenever the defect duration exceeds 36 hours or in the case of alteration of the tissue and full-thickness alteration of the esophageal wall.

We conclude that the use of the diaphragmatic flap is an excellent technique. In the absence of specific contraindications, it is to be preferred to the other pedicle flaps, especially in dealing with early-onset fistulas. It allows fast and safe suture line protection, inducing more rapid healing. Functional modifications are minimal for those patients who underwent esophageal repair and advantageous for those who had pneumonectomy or lobectomy. Apart from the techniques used in primary reclosure, surgeons should use these procedures early because of the high success rate in the presence of a sterile field.

\section{REFERENCES}

1. Petrovsky BV. The use of diaphragm graft for plastic operation in thoracic surgery. J Thorac Cardiovasc Surg 1961;41:348-55.

2. Brewer LA III, Gazzaniga AB. Phrenoplasty: a new operation for the management of pleural dead space following pulmonary resection. Ann Thorac Surg 1968;6:119-26.

3. Westaby S, Shepard MP, Nohl-Oser HC. The use of diaphragm pedicle grafts for reconstructive procedures in the esophagus and tracheobronchial tree. Ann Thorac Surg 1982;33:486-90.

4. Mineo TC, Ambrogi V. Early closure of the postpneumonectomy bronchopleural fistula by pedicled diaphragmatic flaps. Ann Thorac Surg 1995;60:714-5.

5. Goldstraw P, Jiao X. Pericardial repair after extensive resection: another use for the pedicle diaphragmatic flap. Ann Thorac Surg 1996;61:1112-4.

6. Rao KVS, Mir M, Cogbill CL. Management of perforations of the thoracic esophagus. Am J Surg 1974;127:609-12.

7. Avissar E, Sheinfeld A, Lernau O. Repair of esophageal perforation with a diaphragmatic flap. Harefuah 1992;123:22-4.

8. Jara FM. Diaphragmatic pedicle flap for treatment of Boerhaave's syndrome. J Thorac Cardiovasc Surg 1979;78:931-3.

9. Kotsis L, Kostic S, Zubovits K. Multimodality treatment of esophageal disruptions. Chest 1997;112:1304-9.

10. Kotsis L, Agòcs CS. The effectiveness of diaphragmatic pedicled grafts in esophageal injuries and wall reconstruction. Eur J Cardiothorac Surg 1998;14:218-9.

11. Yu YX, Yu KD. Treatment of esophageal achalasia (cardiospasm) with diaphragmatic graft. Twenty-five years' experience. J Thorac Cardiovasc Surg 1989;97:575-7.

12. Fell SC. Surgical anatomy of the diaphragm and the phrenic nerve. Chest Surg Clin North Am 1998;8:281-94.

13. Kalweit G, Feindt P, Huwer H, Volkmer I, Gams E. The pectoral muscle flaps in the treatment of bronchial stump fistula following pneumonectomy. Eur J Cardiothorac Surg 1994;8:358-62.

14. Hammond DC, Fisher J, Meland NB. Intrathoracic free flaps. Plast Reconstr Surg 1993;91:1259-64.

15. Puskas JD, Mathisen DJ, Grillo HC, Wain JC, Wright CD, Moncure AC. Treatment strategies for bronchopleural fistula. J Thorac Cardiovasc Surg 1995;109:989-96.

16. Cauldwell EW, Sickert RG, Lininger RE, Anson BJ. The bronchial arteries: an anatomic study of 150 human cadavers. Surg Gynecol Obstet 1948;86:395-412.

17. Mineo TC, Ambrogi V, Pompeo E, Cristino B, Natali GL, Casciani CU. Comparison between intercostal and diaphragmatic flaps in the surgical treatment of early bronchopleural fistulas. Eur J Cardiothorac Surg 1997;12:675-7.

18. Kamei Y, Moriura S, Ikeda S, Hosokawa H, Hirai M, Naiki K, et al. Combination gastric seromuscolar patch and omental pedicle flap for bronchial fistula. Ann Thorac Surg 1993;56:366-8.

19. Graham DR, Kaplan D, Evans CC, Hind CRK, Donnelly RJ. Diaphragmatic plication for unilateral diaphragmatic paralysis: a 10-year experience. Ann Thorac Surg 1990;49:248-52.

20. Takeda S, Nakahara K, Fujii Y, Minami M, Matsuda H. Plication of paralyzed hemidiaphragm after right sleeve pneumonectomy. Ann Thorac Surg 1994;58:1755-8.

21. Grillo HC, Shepard JAO, Mathisen DJ, Kanarek DJ. Postpneumonectomy syndrome: diagnosis, management and results. Ann Thorac Surg 1992;54:638-51. 